\title{
Brain Imaging of Attentional Networks in Normal and Pathological States*
}

\author{
Diego Fernandez-Duque ${ }^{1}$ and Michael I. Posner ${ }^{2}$ \\ ${ }^{1}$ Rotman Research Institute, Baycrest Centre for Geriatric Care, Toronto, Canada \\ ${ }^{2}$ Sackler Institute, Department of Psychiatry, Weill Medical College of Cornell University, New York, NY, USA
}

\begin{abstract}
The ability to image the human brain has provided a new perspective for neuropsychologists in their efforts to understand, diagnose, and treat insults to the human brain that might occur as the result of stroke, tumor, traumatic injury, degenerative disease, or errors in development. These new findings are the major theme of this special issue. In our article, we consider brain networks that carry out the functions of attention. We outline several such networks that have been studied in normal and pathological states. These include networks for orienting to sensory stimuli, for maintaining the alert state, and for orchestrating volitional control.

There is evidence that these networks have a certain degree of anatomical and functional independence, but that they also interact in many practical situations. Damage to each of these networks, irrespective of the source, produces distinctive neuropsychological deficits. We consider the links between the etiology of the injury and changes in cognition and behavior and examine the role of brain imaging in the study of rehabilitation.
\end{abstract}

Many of the cognitive tasks that humans perform such as reading a word, recognizing an object, or grasping a cup, are very challenging from a computational standpoint. The brain solves this complexity by breaking those cognitive tasks into collections of small computations which are implemented by separate, albeit interacting, brain areas (Posner \& Raichle, 1994). We take the same to be true about the mental operations of the attentional system.

In this paper, we view attention in terms of networks of anatomical areas that carry out the functions of orienting to sensory stimuli, maintaining the alert state, and orchestrating the computations needed to perform the complex tasks of daily life. However, while most of the cognitive networks involve specific domain functions, attentional networks implement a mental function that is domain general. That is, attentional networks carry out energetic and selective control functions that are used regardless of the content of the material. We can attend to the visual domain, but we can also attend to the auditory domain, to the meaning of a stimulus, to our internal thoughts, etc. This domain generality gives attention the ability to influence most, if not all, areas of the brain. For example, imaging studies of the visual system have revealed attentional effects in brain areas specialized for motion perception, color perception, face recognition, and even primary visual cortex (Corbetta, 1998; Corbetta, Miezin, Dobmeyer, Shulman, \& Petersen, 1991, Posner \& Gilbert, 1999). Although attentional effects can be expressed in a multitude of areas, we believe that the source of these influences is limited to a small number of networks.

*This work was supported in part by the grant \#96-47 from the James S. McDonnell Foundation.

Address author correspondence to: Michael Posner, Sackler Institute, Dept. of Psychiatry Box 140, Weill Medical College of Cornell University, 1300 York Avenue, New York, New York 10021, USA. Tel.: (212) 7463781. Fax: (212) 746 5755. E-mail: mip2003@med.cornell.edu 
We argue that the attentional system is anatomically separate from the various data-processing systems that can be activated passively by visual and auditory input (but see Allport, 1993). Attention is neither the property of a single brain area nor is it a collective function of the brain working as a whole. Instead, attention is carried out by discrete anatomical networks, and within each network, specific computations are assigned to different brain areas (Posner \& Petersen, 1990). Although the networks that constitute the attentional system interact with each other, they also have unique aspects.

These claims, as well as the current progress in the anatomy of attention, rely most heavily on two important methodological developments. First, the use of microelectrodes with alert animals has provided evidence for attention-related changes in cellular activity (for a recent review see Chelazzi and Corbetta, 1998). Second, imaging techniques such as PET (positron emission tomography) and fMRI (functional magnetic resonance imaging) have helped to localize cognitive functions in normal human experimental participants (Toga \& Mazziotta, 1994). PET and fMRI can be used together with methods that trace the time course of brain activity in humans by recording electrical and magnetic fields outside the skull, such as ERP (event-related potentials) and MEG (magnetoencephalography) (see Hillyard \& Anllo-Vento, 1998 for a recent review). Methods with good temporal resolution provide a way to trace the rapid time-dynamic changes that occur in the course of human information processing. When combined with the spatial localization of PET and fMRI, it is possible to learn which areas constitute the attentional networks, how those networks interact (i.e., their shared features), and how they are dissociated (i.e., their unique aspects). In doing so, neuroimaging techniques will become a useful tool in the design of rehabilitation procedures that focus on systematic recovery of specific attentional functions.

It is not yet possible to specify the complete attentional system of the brain. However, the last decade has seen great advances in our understanding of the anatomical networks that carry out three major attentional functions: (1) Orienting to sensory stimuli, particularly to locations in visual space (orienting network); (2) achieving and maintaining the alert state (vigilance network); and (3) orchestrating voluntary actions (executive network). Below, we consider each of these attentional functions, describing in turn the normal processing, the networks underlying those processes, deficits found in various pathological states, and evidence for rehabilitation of function.

\section{Orienting}

Orienting refers to the selection of sensory information. Within vision, we usually achieve orienting by directing our eyes toward the location of interest (overt orienting), but it is also possible to assign priority to an area of the visual field without moving the eyes (covert orienting). The function of covert orienting is to assign priority to locations in the visual field. Similarly, eye movements allow those areas to be inspected with the high acuity present for foveal stimuli. Covert attention does not rely upon external forms of orienting, such as head or eye movements. As a consequence, studies on covert attention are particularly useful not only to control for changes in visual acuity, but also to explore exclusively the internal mechanisms of attention.

We start this section by describing the mechanisms of covert orienting in normal vision, after which we review their anatomical substrate. The data suggest a complex network of brain areas that work in an integrated fashion to select areas of interest in the visual field. Later, we consider deficits that may occur in this network due to a wide range of disorders. The major theme will be that many forms of trauma, degeneration, or psychopathology produce problems with orienting, and these problems can best be seen as influencing the normal orienting network in specific ways. Recent studies suggest that deficits of orienting may not be linked so much to etiology (e.g., stroke, closed head injury, Alzheimer's Disease, or schizophrenia), but rather to the specific anatomy of the damage. Finally, we consider efforts to remediate deficits in this network.

\section{Normal Function}

A very simple way to examine covert orienting of attention is to require the person or animal to 
maintain fixation while processing a visual event peripheral to the current fixation. Many studies of normal experimental participants have demonstrated that information is processed more efficiently at an attended peripheral location than at other locations equally distant from the fixation point (Posner \& Petersen, 1990).

In a typical covert orienting task, the experimenter 'cues' the participant's attention to one side or another before the target appears. Attention can be drawn automatically by a peripheral flash (exogenous cueing), or allocated voluntarily by the participant, based on a central arrow that indicates the location where the target is most likely to appear (endogenous cueing). See Figure 1 for an illustration. The participant will be faster to respond to targets at the cued location than to uncued locations, sensory thresholds will be lowered, electrical responses recorded from remote or indwelling electrodes will be enhanced, and blood flow will be increased (for a recent review, see Mangun, Jha, Hopfinger, \& Handy, 1998). Attention thus serves to amplify brain activity at the cued location and produces a behavioral effect of giving priority to that location.

A good way to study orienting under less constrained experimental conditions is with the visual search task. Visual search differs from the covert orienting task in several ways: The spatial selection occurs in a cluttered screen, shifts of attention co-occur with eye movements, there is no exogenous cueing, and the target is specified in terms of a particular object that differs from the background distracters (see Fig. 2 for an illustration).

a.

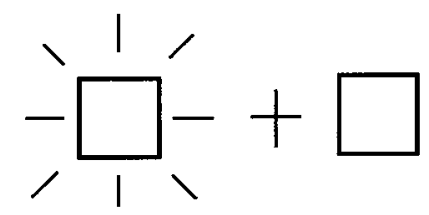

b.

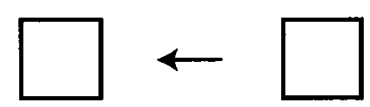

Despite these differences, there is evidence from behavioral, imaging, and clinical studies that both tasks tap similar cognitive functions and neural structures (Luck, Fan, \& Hillyard, 1993). The same cognitive operations are often used to describe both the covert orienting task and the visual search task. In the covert orienting task, invalid trials require a disengagement from the cued location, an attentional movement, and an engagement at the target location, so the target can be detected, and a response decision made. Similarly, in the visual search task, the participant needs to disengage attention from the last location, move attention, and engage it at a new location.

In the visual search task, targets defined by a single pop-out feature (e.g., a red bar among blue distracters) are easily detected in parallel, and detection time does not increase with increased number of distracters in the display, suggesting that feature detection is a pre-attentive process. In contrast, targets defined by the conjunction of two features (e.g., a blue T among red Ts and blue Ls) are more difficult to detect and require serial search. For these targets, detection time increases as a function of the number of distracters in the display, suggesting that the integration of features into objects is an attention-dependent process.

\section{Brain Network}

The anatomical networks involved in orienting are similar for overt and covert orienting as well as for visual search tasks (Corbetta, 1998). PET and fMRI studies show that both covert and overt
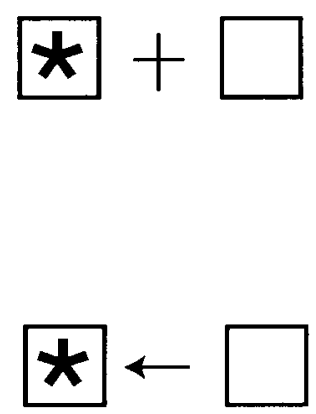

Fig. 1. Upper portion (a.) is a paradigm for studying peripheral attention shifts with exogenous cues at the likely location of the target. Lower portion (b.) uses an arrow (far left) to direct attention either to the cued location or to an uncued location. 


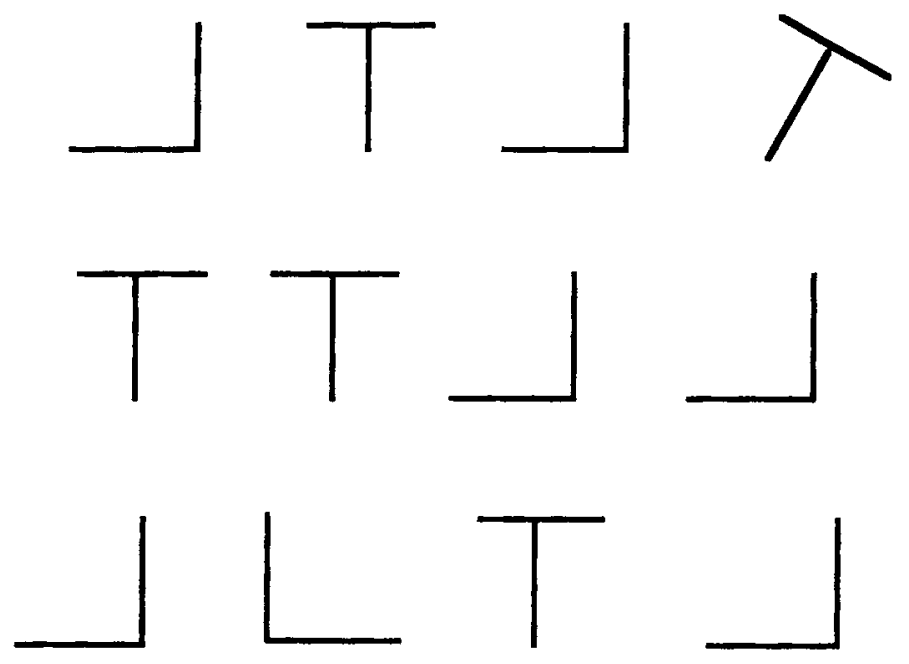

Fig. 2. The tilted $\mathrm{T}$ pops out and pulls attention in a manner similar to an exogenous cue. The $\mathrm{L}$ target, however, requires an effortful search to separate the target from the distracters.

orienting activate the precentral gyrus of the frontal lobe and areas in the parietal lobe (Corbetta, 1998; Rizzolatti \& Craighero, 1998). Data from animal work provide further support for common neural structures underlying overt and covert orienting, such as the parietal cortex (areas LIP and $7 \mathrm{a}$ ), the frontal eye fields and some subcortical structures, including the superior colliculus and the thalamus (Andersen, Brotchie, \& Mazzoni, 1992; Mazzoni, Bracewell, Barash, \& Andersen, 1996).

As we mentioned earlier, attention can be drawn automatically to a location by an exogenous cue, or can be voluntarily allocated by the experimental participant based on the information of an endogenous cue. Imaging studies reveal that exogenous and endogenous cues activate similar areas both in frontal and parietal cortex, favoring the existence of a general neural system for visuospatial attention that can be used by automatic as well as voluntary cues (Corbetta, Miezin, Shulman, \& Petersen, 1993; Nobre et al., 1997; but see Làdavas, Menghini, \& Umiltà, 1994).

The anatomical overlap between overt and covert orienting tasks, as well as the overlap between exogenous and endogenous cueing, provide support for the existence of a common neural system of eye movements and orienting. However, the orienting network is also activated dur- ing attention tasks that are unrelated to eye movements. For example, when experimental participants shift attention between two dimensions, such as color and shape, there is bilateral increased activation in the superior parietal cortex, suggesting that the parietal cortex is involved in attention shifts even when those shifts are nonspatial (Le, Pardo, \& Hu, 1998). Cueing attention to a specific time interval during which the target is most likely to occur activates the intraparietal sulcus, even in the absence of any spatial cue (Coull \& Nobre, 1998). These results argue in favor of a supramodal orienting system, one that can select information based not only on spatial location but also on other, non-spatial features, such as color or time.

\section{Orienting Deficits}

One of the most common abnormalities of orienting is a strong lateral bias in which experimental participants have difficulty disengaging from a cue presented on the side of the lesion to respond to a target located on the side opposite of the damage. In the extreme form of such "neglect," patients may miss entirely stimuli on the side opposite to the lesion. In a less extreme form called "extinction," they miss contralesional stimuli only when presented at the same time as an ipsilesional event (for a review, see Rafal, 
1998). In patients for whom the damage is slight, this difficulty may only show up as an abnormally slow reaction time in disengaging attention from the ipsilesional field. This disengagement deficit has been found, using the covert orienting task, in clinical populations suffering from stroke, Alzheimer's Disease, and schizophrenia, as well as in reversible lesion studies in normal participants, and in pharmacological and lesion studies in monkeys.

\section{Stroke Patients}

It has been known for over a century that patients with strokes of the parietal lobe neglect locations in the field opposite the lesion. Research dating back to the 1980s has demonstrated that patients with parietal damage have a very distinct and virtually permanent deficit, as revealed in the covert orienting task (Morrow \& Ratcliff, 1987; Posner, 1988). Once their attention is oriented to the stimulus on the side of the lesion, these patients show a delay in reacting to events on the side opposite the lesion. The deficit is not sensory because their performance is normal or near normal if attention is not first drawn to the side of the lesion. Yet the deficit can be quite profound and targets are often completely missed at the unattended location. This finding suggests that the parietal lobe is responsible for the mental operation of disengaging from a cue to respond to a target in the neglected side.

Lesions of the superior colliculus and thalamus also produce deficits in covert orienting, but of a different kind. The collicular lesions are identified with the act of moving attention, while the thalamus is thought to be part of a pathway by which frontal and parietal areas might make contact with the ventral pathway involved in object recognition. The pulvinar nucleus of the thalamus is associated with the ability to engage attention in an object. These results suggest that each of the components of orienting (disengagement, move, engagement) has a distinct anatomy, and that different behavioral deficits could be brought about by specific lesions of the neural structures underlying each component.

The cueing task and the theoretical approach of breaking down orienting into three anatomically distinct computations have made it possible to study an act of attention in normal humans, in a wide variety of patients, and in alert monkeys. Of course, this approach also has limitations. For example, the cueing task fails to consider the competition provided by the non-target elements, an important aspect of attention that is emphasized in visual search tasks. Also, it has been possible to model the deficits found in neglect patients by considering the imbalance between the hemispheres created by the lesion, leading some to question whether it is necessary to postulate special mental operations (Cohen, Romero, Servan-Schreiber, \& Farah, 1994; Farah, 1994). The competition model is favored by some studies of cellular recording in alert monkeys that required them to attend to target locations (Desimone \& Duncan, 1995). Finally, while bilateral lesions of the parietal lobes produce a difficulty in dealing with two simultaneous objects (Balint's syndrome), there is little evidence that this is due to an inability to disengage (Robertson, 1998). Despite its limitations, the disengagement hypothesis has made it possible to investigate the contribution of the parietal lobe to covert orienting across a number of different pathologies.

\section{Degeneration}

Patients with Alzheimer's disease (AD) may show an initial difficulty in orienting. Such patients show a behavioral profile in orienting to endogenous cues similar to that of patients with parietal lesions (Buck, Black, Behrmann, Caldwell, \& Bronskill, 1997; Parasuraman, Greenwood, Haxby, \& Grady, 1992). That is, they have a difficulty in responding to trials in which the target occurs at the uncued location (i.e., disengagement deficit). Moreover, PET studies reveal low metabolic activity in the parietal cortex at early stages of AD (see Parasuraman \& Greenwood, 1998 for a review).

In $\mathrm{AD}$, there is a known deficit in central cholinergic activation, and the parietal dysfunction in $\mathrm{AD}$ is likely to be secondary to such cholinergic deficit (for a review see Lawrence \& Sahakian, 1995). Interestingly, a lesion of the basal cholinergic system of monkeys leads to a behavioral profile in the covert orienting task identical to that of patients with $\mathrm{AD}$ and neglect patients (Voytko 
et al., 1994). Similar results have also been found after selective cholinergic lesions in rats (Chiba, Bushnell, Oshiro, \& Gallagher, 1999).

The parietal cortex is involved not only in covert orienting but also in overt orienting (i.e., eye movements). In this case as well, patients with $\mathrm{AD}$ evidence a profile consistent with a disengagement impairment: Their gaze responses are slow and they have a tendency to make perseverative errors (Scinto et al., 1994).

Other deficits in patients with $\mathrm{AD}$, such as poor performance in a cancellation task, are consistent with parietal lesion (Foldi, Jutagir, Davidoff, \& Gould, 1992). Patients with AD also have abnormally high slopes for visual search, that is, as the number of distracters is increased, reaction times (RT) become increasingly slower than normal (Nebes \& Brady, 1989, but see Greenwood, Parasuraman, \& Alexander, 1997). This pattern reveals patients' inability to search for items, consistent with an inability to disengage attention from distracter locations. While some patients with $\mathrm{AD}$ have impaired spatial attention, their ability to select items based on color or other non-spatial features seems normal. Patients with AD also demonstrate a relatively normal alerting effect to warning signals (Nebes \& Brady, 1993).

Although AD eventually affects many mental functions, cognitive deficits in AD may not arise as a global loss, but as the selective disruption of particular neural networks and the cognitive functions they subserve (Schwartz, 1987). Here we have illustrated the principle as it applies to attentional functions, but it is also likely to apply to other mental functions that are affected in AD. For example, episodic memory deficits, which are apparent in the early stages of the disease, correlate in highly specific ways with the early onset of medial temporal lobe atrophy (Köhler et al., 1998)

\section{Transmitter Deficits}

The nucleus basalis is the source of cholinergic modulation of many cortical areas, including the parietal lobe. Lesion studies in monkeys reveal that when the nucleus basalis is lesioned, experimental participants have a specific difficulty in disengaging attention from an ipsilesional cue to engage a target on the side opposite to the lesion (Voytko et al., 1994). Davidson and Marrocco
(2000) applied systemic and local injections of scopolamine, a muscarinic antagonist of acetylcholine, to areas of the parietal lobe previously shown to be actively involved in orienting. Scopolamine served to slow the ability to orient toward the cue without changing the ability of the warning cues to improve RT, suggesting that the cholinergic system is involved in spatial orienting, with little influence on the response to warning signals.

\section{Psychopathology}

It is common to view schizophrenia as a higher order attentional deficit disorder (e.g., Frith, 1992). However, the emergence of neuroimaging technology has allowed the study of specific links between the cognitive deficits observed in schizophrenia and its anatomical substrates (Gur \& Pearlson, 1993).

PET studies of never-medicated patients with schizophrenia show evidence of a left globus pallidus (basal ganglia) abnormality (Early, Posner, Reiman, \& Raichle, 1989a, 1989b). As the disorder progresses, there appears to be a widespread reduction of flow and metabolism in the frontal lobes involving both the anterior cingulate and the dorsolateral prefrontal cortex.

Never-medicated first break schizophrenics show an abnormality in the shifting of visual attention. This spatial deficit involves a specific difficulty in shifting attention toward the right visual field, while attention shifts toward the left visual field are normal (Posner, Early, Reiman, Pardo, \& Dhawan, 1988). This deficit is very specific because it is limited only to one visual field and cannot be due to an inability to understand instructions or to lack of motivation. A number of studies using chronic patients have failed to replicate this finding, but a study testing patients at different stages of their disease has reconciled these inconsistencies (Maruff, Currie, Hay, McArthur-Jackson, \& Malone, 1995). Unmedicated first break schizophrenics who were acutely psychotic showed an asymmetry in shifting attention to targets in the right visual field. This asymmetric spatial attention deficit was partially resolved with brief periods of medication and was completely gone in chronic patients (Maruff et al., 1995). The deficit is absent in monozygotic twins of patients with schizophrenia who do not 
show the disorder (Pardo et al., 2000). Thus, the attentional deficit appears to be a part of the early left hemisphere malfunction found in acute psychotics.

The PET studies of never-medicated schizophrenics suggest a frontal lobe deficit, but not a parietal deficit. In accord with this anatomy, the deficit in schizophrenia appears to result from an abnormality in executive attention. When normal experimental participants are asked to verbally shadow auditory messages, thus tying up the frontal executive attention network, they show an abnormality similar to that found in patients with schizophrenia (Posner, 1988). This finding, together with the clear evidence that acute schizophrenics have a distinct fronto-temporal abnormality of language, suggests that frontal control structures are involved in this disorder. The executive attention network seems to play a role in the initiation of the attentional shifts, and even in the absence of posterior parietal lesions, deficits of the executive network may interact with the parietal areas to produce abnormalities of orienting.

\section{Reversible Lesions}

Attentional deficits, frequently seen in clinical populations, have also been reproduced by artificially lesioning attentional areas in normal experimental participants. This is done by applying brief magnetic pulses to the head overlaying the relevant cortex (Transcranial Magnetic Stimulation, TMS; Walsh \& Cowey, 1998). As we have described, visual extinction to simultaneous stimuli is a frequent sign of neglect, while neglect patients are usually capable of perceiving a contralesional stimulus when it is presented in isolation. Similarly, repetitive pulses of TMS to the parietal cortex impair detection of a contralateral stimulus during bilateral stimulus presentation (extinction-like effect) but not during unilateral stimulus display (Pascual-Leone, Gomez-Tortosa, Grafman, \& Alway, 1994). TMS to the right parietal cortex also disrupts attention-dependent visual searches, such as a search for a target defined by the conjunction of two features (e.g., a blue $\mathrm{T}$ among red $\mathrm{Ts}$ and blue Ls), but has no influence on pre-attentive searches (Ashbridge, Walsh, \& Cowey, 1997).

\section{Summary}

The studies described in this section reveal that a wide variety of disorders influence the brain's sensory orienting system by making shifts of attention difficult. Much of neuropsychology has involved the study of particular disorders, and an overarching principle has been that remediation depends upon the cause of the deficit. In contrast, the literature reviewed in this section suggests that the behavioral deficit might be closely linked to the dysfunction of a brain structure (e.g., the parietal lobe on one side) irrespective of whether the cause is a sudden onset like a stroke or a slow change as in Alzheimer's Disease. Which brain area is damaged appears to be more important for producing a disengagement deficit than the specific cause of the damage. Stroke and Alzheimer's disease are often disorders of the elderly, but evidence from normal participants undergoing TMS suggests that the age of onset may also be of less importance than the brain network affected. The schizophrenia data show that in the absence of posterior lesions, deficits of other attentional networks that interact with the parietal system may also produce abnormalities of orienting.

\section{Rehabilitation}

For a long time, neglect rehabilitation was considered of little therapeutic interest, because there is a great deal of spontaneous recovery from strokes that produce neglect. This view has changed in the last ten years, as more careful assessments using covert orienting and visual search tasks have revealed persistent attention deficits in neglect patients and deficits in other types of disorders (Robertson, 1999; Robertson, Halligan, \& Marshall, 1993).

The strategies for neglect rehabilitation have been largely influenced by theories on the relation between covert and overt orienting, the imbalance between hemispheres, and the existence or not of separate systems for voluntary and automatic orienting.

\section{Overt and Covert Orienting}

Imaging studies provide compelling evidence for the interdependence of covert and overt orienting. If attention shares neural structures with eye 
movement, the stimulation of eye movement reflexes toward the neglected side should ameliorate neglect. A number of studies have investigated this possibility. Rehabilitation with vestibular stimulation (Cappa, Sterzi, Vallar, \& Bisiach, 1987; Vallar, Sterzi, Bottini, Cappa, \& Rusconi, 1990), or with optokinetic nistagmus (Pizzamiglio, Frasca, Guariglia, Incoccia, \& Antonucci, 1990), both of which produce a reflexive eye deviation toward the neglected side, yield a reduction of neglect. However, this benefit is short-lived. Activation of the superior colliculus via the retino-tectal path by a dynamic stimulus reduces neglect more than a static stimulus, but this amelioration is also short-lived (Butter, Kirsch, \& Reeves, 1990).

\section{Competition between Hemispheres}

Some theorists propose that attention is an emergent property of the competition between visual stimuli for various neural systems (e.g., Desimone \& Duncan, 1995). This view is consistent with the increased difficulty in visual search tasks as a function of the number of objects present in the visual field. One account postulates that a balance exists between the two hemispheres in the normal brain, with each hemisphere competing with the other for stimulus processing, and that neglect occurs when one hemisphere is lesioned, and an imbalance follows (Kinsbourne, 1977). As a consequence of such an imbalance, perception of a contralesional stimulus is impaired but perception of an ipsilesional stimulus is enhanced above normal levels. Evidence for this supranormal sensitivity to ipsilesional stimuli comes from studies in which neglect patients outperform controls in the detection of a stimulus when the stimulus is presented in the right visual field (ipsilesional field; Làdavas, Pretronio, \& Umiltà, 1990). Similarly, reversible lesions of the parietal lobe with TMS decrease sensitivity to a contralateral somatosensory stimulus, but increased sensitivity to an ipsilateral stimulus (Seyal, Ro, \& Rafal, 1995).

The idea of hemispheric competition has been extended to rehabilitation studies, with the notion that inhibitory competition from undamaged circuits, such as the contralateral hemisphere, may impede the recovery of function. In one study, the contralesional superior colliculus was deprived of direct retinal inputs by patching the ipsilesional eye. The hypothesis was that, in the absence of competition from the normal hemisphere, the lesioned areas would reactivate (Butter et al., 1990). Although the procedure did reduce neglect, the recovery was incomplete, short-lived, and did not generalize to other tasks.

In other rehabilitation studies, patients with right parietal lesions ameliorated their neglect by moving their left hand in the left hemispace (Robertson \& North, 1993, 1994). The benefit lasted several weeks after training, and generalized to everyday function (Robertson, North, \& Geggie, 1992). Importantly, bilateral activation abolished the benefit, suggesting that competition from the healthy hemisphere had detrimental effects on the rehabilitation of the lesioned hemisphere. This is a situation that resembles the phenomenon of extinction, in which neglect patients are aware of an object in the contralesional field when presented alone, but not when presented in synchrony with another object in the ipsilesional field. Despite this evidence, the question of the mechanism by which using a contralesional limb ameliorates neglect remains open. One possibility, consistent with the competition hypothesis, is that using the contralesional (left) arm activates the lesioned hemisphere (right). The other possibility, consistent with an attentional network hypothesis, is that using the contralesional arm cues attention toward the contralesional field. There is some evidence in favor of the latter possibility. Specifically, patients using their contralesional (left) hand demonstrate less neglect only if they start the task in the contralesional (left) hemispace. When they start in the ipsilesional (right) hemispace, they show substantial neglect even when using the contralesional (left) arm. In this condition, the activation of the lesioned hemisphere by motor activity of the contralesional limb is present, but the spatial cueing toward the contralesional field is absent, suggesting that the cause of improvement is the attentional cueing, not the hemispheric activation of the lesioned hemisphere (Halligan, Manning, \& Marshall, 1991).

Minimizing contralateral competition is beneficial not only for attention rehabilitation but also for the rehabilitation of other systems, such as the 
motor system. For example, preventing movement of the unaffected limb leads to improvement in a paretic limb (Taub et al., 1993), and intensive behavioral training of the affected hand can minimize the loss of brain areas surrounding the stroke (Nudo, Wise, Fuentes, \& Milliken, 1996). Thus, the competition between hemispheres might be a domain general principle guiding plasticity in motor as well as attentional systems.

\section{Automatic and Voluntary Orienting}

There is strong evidence that cueing neglect patients to the side opposite the lesion helps their performance (Humphreys \& Riddoch, 1993). However, the mechanism underlying this effect is a matter of debate. Imaging studies reveal that exogenous and endogenous cues activate similar areas in frontal and parietal cortex, thus favoring the existence of a general neural system for visuospatial attention that can be used by automatic as well as voluntary cues (Corbetta et al., 1993; Nobre et al., 1997; for a review see Corbetta, 1998). In contrast, some researchers have proposed the existence of two separate orienting systems, a voluntary orienting system which responds to endogenous symbolic cues and involves the frontal lobes, and a separate automatic orienting system, which responds to exogenous cues involving parietal areas (Làdavas, 1993). Support for this idea comes from clinical studies in which lesions of the frontal lobes lead to deficits in endogenous (voluntary) orienting, whereas parietal lesions selectively impair exogenous (automatic) orienting (Làdavas, 1993).

If endogenous orienting depends on a different neural system than exogenous orienting, it should be possible to train the unimpaired system (i.e., frontal) to compensate for the deficit in the impaired system (i.e., parietal). Làdavas and colleagues (1994) addressed this possibility in a rehabilitation study in which twelve patients with right parietal damage and neglect syndrome practiced an endogenous orienting task, either moving both their eyes and their attention toward the cued location (overt condition), or moving their attention while keeping the eyes fixated on the center (covert condition). A group of neglect patients who did not participate in the training served as a control group. Training improved overall perfor- mance, and also diminished the visual field asymmetry (a measure of neglect) beyond spontaneous recovery. Moreover, training improved the ability to attend to cues in the neglected left field. Thus, it seems that the training of the voluntary orienting system helped participants to overcome their rightward bias, and to cue attention toward the left visual field (Làdavas et al., 1994).

A similar distinction between endogenous and exogenous shifts has been suggested for overt orienting, with the existence of separate neuroanatomical structures responsible for voluntary and reflexive saccades (LaBerge, 1995; Maunsell, 1995; Schall, 1995). Consistent with this view, patients with frontal lobe lesions have difficulty producing voluntary saccades to locations opposite to the automatic exogenous cue (Guitton, Bouchtel, \& Douglas, 1985; Làdavas, 1997; Rivaud, Muri, Gaymard, Vermersch, \& PierrotDeseilligny, 1995). Finally, artificially 'lesioning' the prefrontal cortex with TMS impairs saccades in response to endogenous (voluntary) cues, but not to exogenous (automatic) cues (Ro, Henik, Machado, \& Rafal, 1997).

\section{Vigilance}

Vigilance is the ability to achieve and sustain the alert state. In general, two types of tasks have been used to study vigilance: warning tasks and continuous performance tasks. Tasks using a warning signal have tested how quickly a person can obtain maximum alertness. The reaction to a warning signal, particularly if it is auditory, has a strong automatic component (exogenous alerting). Readiness can also be achieved by an incentive to process an expected target (endogenous alerting). Continuous performance tasks have studied the sustained attention of vigilance by requiring participants to remain prepared to detect relatively rare and generally rather weak targets or by using a task that requires continual responding. In order to perform these tasks, participants must remain alert and avoid processing any irrelevant information so that stray thoughts do not distract them from the target.

Both warning signal and continuous performance tasks require high levels of alertness for optimal performance and both have been shown to be influenced by similar processes. Several 
years ago, Posner and Petersen (1990) published an extensive review of studies involving reaction time of normal experimental participants, parietal patients, and split brain patients. The results converged to reveal that right frontal and parietal areas were of particular importance for obtaining and sustaining the alert state. Since that time, imaging and patient data have generally confirmed this finding, and studies with alert monkeys and normal participants have hinted at which transmitter systems may be involved.

\section{Brain Network}

A vigilant state increases activation in the right frontal-parietal system, even when no stimulus actually occurs (Pardo, Fox, \& Raichle, 1991). In this study, participants monitored for two minutes any change in the intensity of a fixation mark that in fact remained invariant. Similar results occur when participants monitor one of their toes for a tactile stimulus that is never presented. The activation is lateralized to the right hemisphere independent of whether the right or the left big toe is being monitored. Auditory vigilance tasks also activate right frontal areas (Belin et al., 1998; Cohen, Semple, Gross, \& Holcomb, 1988). Moreover, activation in those areas decreases as a function of time, and this decrement correlates with some measures of vigilance decrement, such as the slowing down in RTs (Paus et al., 1997). These results reveal the existence of a sustained attention system that is anatomically separate from the data-processing systems.

Sustained attention is a central aspect of many cognitive functions, and several studies have explored the neural bases of such interactions. For example, Coull and colleagues used a variant of the rapid visual information processing task, together with PET, to study activations related to sustained attention and working memory (Coull, Frith, Frackowiak, \& Grasby, 1996). In this task, digits are rapidly presented one at a time and participants have to detect target sequences of two or three digits. This task requires not only sustained attention, but also selective attention, because successful performance requires participants to attend only to items that are part of the correct sequence. Successful performance also requires working memory. This task, as well as a variant without the memory requirement (detection of a target digit), activates the frontal-parietal system, with the memory component most likely activating the left side and the sustained attention component tapping mostly the right side.

\section{Vigilance Deficit}

Clinical data similarly suggest that right frontal lesions impair patients' ability to voluntarily sustain attention. When asked to count a series of infrequent tones, patients with right frontal damage perform worse than patients with left frontal damage (Wilkins, Shallice, \& McCarthy, 1987). In continuous performance tasks, the error increment over time is larger for right than for left frontal patients, suggesting that right frontal lesions not only make experimental participants less vigilant, but also accentuate the vigilance decrement over time (Rueckert \& Grafman, 1996). Right lesions seem to produce a deficit in internally generated alertness, as patients are unable to sustain their own attention.

Alertness can be increased by internally generated signals but also can be increased automatically by the presence of an external stimulus, even if it is uninformative about the future occurrence of a target (Robertson, Mattingley, Rorden, \& Driver, 1998). While the frontal lobe is a likely generator of endogenous alerting signals, the external stimulus exercises its alerting effect by ascending thalamic projections.

Both the voluntary, effortful endogenous warning effect, and the automatic, effortless exogenous effect likely act upon the right parietal lobe which, as described in previous sections, is also part of the orienting system. Lesions of the parietal lobe influence both alerting and orienting functions, and patients with right parietal damage show not only deficits in maintaining the alert state, but also in shifting attention.

Both deficits in alertness and in orienting appear to contribute to clinical neglect. For example, an uninformative alerting cue reduces neglect to stimuli presented in the left visual field. This effect is not merely a motor effect, as it is evident when patients are asked to compare the onset of a right and a left event (temporal order judgment task). Under baseline conditions, when visual items are presented simultaneously, neglect 
patients with right parietal lesions perceive the left items as having a later onset. This illusion occurs because their attention to the left side is reduced, and as a consequence the visual processes are slower to reach awareness in that hemifield. However, when the target is preceded by an auditory warning signal, this bias is eliminated, suggesting that phasic alertness is beneficial in reducing neglect (Robertson et al., 1998).

\section{Transmitters}

Studies of alert behaving monkeys have shown that the readiness induced by warning signals can be blocked by drugs that reduce noradrenergic cell firing, such as clonidine and guanfacine (Marrocco \& Davidson, 1998; Witte \& Marrocco, 1997). Clonidine increases overall RT and guanfacine decreases it, but most importantly, both drugs effectively block the warning effect of a preceding cue. In contrast, the ability to orient toward the cue is unaffected by these drugs. Related work in humans further suggests that clonidine slows overall RT, but in contrast to research with monkeys, aspects of orienting are also affected (Clark, Geffen, \& Geffen, 1989). These results support the idea that the transmitter noradrenaline mediates the alerting effect.

There is evidence that noradrenaline is important not only for achieving the alert state, but for sustaining attention over time. Performance in a sustained attention task by alert monkeys is dependent on the activation of noradrenergic neurons in the locus coeruleus. Targets in a continuous performance task are more likely to be missed when they fail to activate the noradrenergic response (Aston-Jones, Rajkowski, Kubiak, \& Alexinsky, 1994). In humans, clonidine decreases sustained attention leading to more attentional lapses. Moreover, this effect is reversed by clonidine blockers such as iadoxan, as well as by increases in environmental arousal such as loud noises (Smith \& Nutt, 1996). Clonidine also impairs performance in a rapid visual information processing task, and this effect is most pronounced during low arousal states (Coull, Robbins, Middleton, \& Sahakian, 1995).

Anatomically, both orienting and alerting appear to be partially implemented in the right parietal lobe. However, a behavioral study that examined both processes suggests that their contributions to overall reaction time are independent (Fernandez-Duque \& Posner, 1997). It is remarkable that the two processes share a relatively common anatomy within the posterior parietal lobe, but involve different transmitters and thus are dissociable at both the cognitive and synaptic levels.

\section{Rehabilitation Studies}

Some recent studies have tried to rehabilitate specific attentional networks (Kinsella, 1998; Sohlberg, McLaughlin, Pavese, Heidrich, \& Posner, 2000; Strum, Willmes, Orgass, \& Hartze, 1997). These studies suggest that rehabilitation procedures should focus on the particular attentional operations of the lesioned area, while at the same time considering the contribution of those deficits to other attentional functions.

In one study (Strum et al., 1997), a computerized rehabilitation program was designed to try to enhance specific attentional networks. Training tasks were designed that exercised four aspects of attention: phasic alertness, vigilance, selective attention, and divided attention. Phasic alertness and vigilance are both related to what we call the vigilance network, while selective attention is related to the orienting network, and divided attention is related to the executive network. Before and after training, patients' attentional abilities in each domain were tested using other tasks to prevent specific practice effects. The results revealed a hierarchical organization of attentional functions. Specific training in vigilance or alertness improved these capacities as well as selective and divided attention. Selective attention training improved selective attention and divided attention, but not alertness. Training in divided attention only improved divided attention capabilities. The authors concluded from these findings that vigilance and alertness are the most fundamental functions in the hierarchy, and that selective attention and divided attention recruit these functions for their normal operation.

Another study that utilized a practice-oriented therapy (attention process therapy) for patients with brain injury showed a therapy specific overall improvement in executive attention tasks, but some tasks only improved for patients with relatively high vigilance scores and not for patients 
who were deficient in vigilance (Sohlberg et al., 2000).

A third rehabilitation study tested the possible interaction between vigilance and orienting by training patients to increase their self-alertness, and exploring whether the rehabilitation of selfalertness had an impact on patients' neglect (i.e., orienting deficit; Robertson, Tegnér, Tham, Lo, \& Nimmo-Smith, 1995). Exogenous alertness was used as a basis for training patients to selfalert. External warning signals were presented, and patients were instructed to generate a selfalertness signal in response to it. Exogenous alertness, as produced by a loud noise, depends on a thalamo-mesencephalic path and is relatively unimpaired in patients with right parietal damage. After the training procedure was explained, the patient started the task and at variable intervals the experimenter knocked on the table while at the same time saying "Attend!" in a loud voice. At the next stage in the training, it was the patient who shouted "Attend!" each time the experimenter knocked on the table. Later, the patient would do both the knocking and the vocal command, first loudly, then subvocally, and finally mentally. Patients were encouraged to try this self-alertness method in their everyday life. This rehabilitation training not only improved patients' self-alertness, but also reduced the extent of their spatial neglect.

Taken together, these findings from rehabilitation studies suggest that rehabilitation procedures need to take into consideration possible vigilance deficits. More generally, they reveal the benefits of systematically analyzing interactions and dissociations of the attentional networks, instead of treating attention as a monolithic concept. They also demonstrate that behavioral therapy can be successful in improving vigilance skills.

\section{Executive Attention}

Broadly speaking, executive attention involves effortful control or coordination, particularly in tasks in which the response is not fully determined by the stimulus (Norman \& Shallice, 1986). The executive system participates in task switching (Hayes, Davidson, Keele, \& Rafal, 1998; Rogers \& Monsell, 1995), inhibitory control (Casey, in press), conflict resolution, error detection, and allocation of attentional resources (for reviews, see Bush, Luu, and Posner, 2000; Carter, Botvinick, and Cohen, 1999; Posner and Rothbart, 1998). It participates in planning, and it involves efforts to process novel stimuli and to execute novel actions (Posner \& DiGirolamo, 1998).

This definition of executive attention is admittedly broad, and in recent years researchers have tried to break these executive processes into smaller, more tractable, units of analysis. However, what those units might be, as well as which anatomical areas implement them, remains a matter of debate.

\section{Brain Network}

Neuroimaging studies have shown activation of a network of brain areas in tasks that require experimental participants to deal with conflict, error, or novelty, or that demand other kinds of effortful cognitive processing (Bush et al., 1998; Posner $\&$ DiGirolamo, 1998). The areas usually activated include the anterior cingulate and supplementary motor area, the orbitofrontal cortex, the dorsolateral prefrontal cortex, and portions of the basal ganglia and the thalamus.

Due to space limitations, we will only review the role of the anterior cingulate, but it should be stressed that the other areas may be of equal importance. The Stroop task has frequently been used to explore the role of the anterior cingulate in conflict resolution. In this task, a colored word is presented, and the participant is instructed to ignore the meaning of the word and respond to the color of the ink (e.g., green ink). The word may be congruent with the ink color (e.g., word 'green'), incongruent (e.g., word 'red') or neutral (e.g., word 'dog'). In Stroop and Stroop-like tasks, participants have to overcome a prepotent response (i.e., responding to the word itself) to provide the correct answer (i.e., responding to the ink color).

The anterior cingulate participates not only in cognitive regulation, but also in emotional regulation. A meta-analysis of studies using Stroop-like tasks suggests that ventral areas of the anterior cingulate and the orbital frontal cortex are closely involved with emotional regulation, whereas dorsal areas of the anterior cingulate are recruited for 


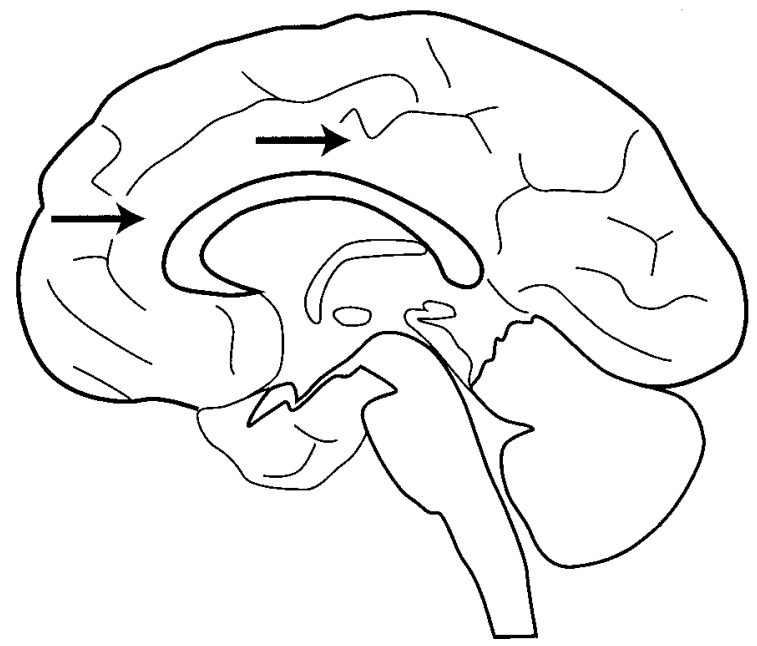

Fig. 3. Ventral portions of the anterior cingulate have been active in various emotional tasks (bottom arrow) while more dorsal portions have been active in the Stroop and other cognitive tasks (top arrow). Adapted from Bush et al., 1998.

purely cognitive tasks (see Fig. 3 adapted from Bush et al., 1998 for an illustration).

Posner and Rothbart (1998) have developed a perspective on why the anterior cingulate might be involved in both emotional and cognitive self-regulation. They argue that in infancy, the organism is faced with the difficult problem of controlling distress and other forms of negative emotion. Since distress is widely thought to involve the amygdala, it seems plausible that the anterior cingulate, which is the outflow of the limbic system, would be involved in its control. In support of this view, adults show activity in the anterior cingulate when exposed to pain. Moreover, the activation in the anterior cingulate, but not in other areas involved in pain, reflects the degree of felt distress when participants are taught to control their pain responses. This suggests that anterior cingulate activation is related to efforts to control the pain (Rainville, Duncan, Price, Carrier, \& Bushnell, 1997). According to Posner and Rothbart (1998), when the child is faced with the need to selectively regulate cognitive activity in conflict tasks, the same brain system already heavily involved in self-regulation could serve as an appropriate vehicle. In support of this view, efforts at self-regulation of cognitive activity appear to correlate with negative affect in 3- to 4-year-olds (Posner \& Rothbart, 1998). For example, performance on compatible and incompatible Stroop-like tasks is related to parental reports of their children's frustration reactions. Further support for this link between cognitive and emotional self-regulation has come from studies of children ages 7 to 16 , showing that the size of the anterior cingulate correlates with the reaction time needed to carry out attention demanding tasks, even when controlling for age differences. In contrast, reaction time in simpler routine tasks involving little attentional control is unrelated to cingulate size (Casey et al., 1997).

Further evidence for the role of the anterior cingulate in the regulation of emotion and cognition comes from studies of error detection. For example, the anterior cingulate is activated in normal adults following error detection or negative feedback (see Carter et al., 1999; Luu, Collins, $\&$ Tucker, 2000). The error-dependent activity appears to be related to the negative experience of having made a mistake, because there is a significant correlation between cingulate activity during error detection and the personality trait of negative emotionality (Luu, Collins, \& Tucker, 2000). In children, the degree of cingulate activity during difficult task performance is also related to the likelihood of error (Casey et al., 1997). 
Despite the evidence revealing that both emotion and cognitive tasks activate the anterior cingulate, it is unknown whether the anterior cingulate is an integrated cognitive-emotional system, or a set of independent modules. A certain amount of structural differentiation exists (Badgaiyan \& Posner, 1998; Devinski, Morrel, \& Vogt, 1995). On the other hand, the possibility of an integrated set of subsystems in which emotion and cognition converge is consistent with the idea that emotions are an integral part of cognitive life, as well as with the extensive literature linking emotion to cognition. In summary, it is likely that different areas of the anterior cingulate perform distinct, albeit interacting, executive functions.

\section{Deficits of Executive Function}

Impairments of the executive system have been reported in a variety of normal and pathological states that involve frontal areas, including aging (West, 1997), traumatic brain injury (Strum et al., 1997), frontal strokes (Duncan, 1986), dementias (Parasumaman \& Greenwood, 1998), schizophrenia (Frith, 1992), and attention deficit disorder (Barkley, 1998). Disruptions of corticostriatal loops, such as those evidenced in Parkinson's Disease, also lead to deficits in executive processes.

As described in the previous section, an important aspect of executive control is the ability to inhibit a prepotent or habitual response. In everyday life, the failure to inhibit these responses is revealed as a slip of action. Patients with traumatic brain injury commit an increased number of slips of action (Robertson, Manly, Andrade, Baddeley, \& Yiend, 1997). This failure in inhibitory control may be a primary executive deficit, or may be secondary to a vigilance deficit, which is usually impaired in patients with closed head injury due to their lesion in frontal areas. To explore this question, Robertson et al. (1997) developed a version of the continuous performance task in which participants were required to press the key every time a frequent non-target occurred, and to inhibit this response when an infrequent target appeared. Robertson and colleagues predicted that an inability to maintain the alert state would lead to an increase in commis- sion errors. Consistent with this view, the number of commission errors was correlated with attentional failure in everyday life situations, as assessed by a questionnaire. The correlations occur both in normal experimental participants and in patients with traumatic brain injury, but for the patients, errors and everyday life attentional failures were more frequent. These results are consistent with the hypothesis that vigilance and inhibitory control interact.

Other executive functions, such as error detection, also seem to be modulated by sustained attention. After making an error in the continuous performance task, normal participants slow down, presumably in an attempt to increase accuracy. In contrast, patients with traumatic brain injury who have impaired vigilance fail to slow down after an error, as if they had failed to detect the error, or to modify performance to minimize future errors (Robertson et al., 1997).

Another important aspect of executive control is the ability to coordinate multiple tasks into a coherent sequence of actions. The dual task paradigm is often used to test this ability. In a typical dual task paradigm, a pair of letters appears on one side of the screen, and participants have to make a speeded response to the location of the stimulus, followed by an unspeeded response to the identity of the letters. Although the identity task is unspeeded and occurs after the primary task, it nevertheless produces a cost to the primary response. This cost is presumably due to the coordination of the two responses (Umiltà, Nicoletti, Simion, Tagliabue, \& Bagnara, 1992). Patients with traumatic brain injury, who are thought to have frontal lobe lesions, show an increased cost in dual task performance (Umiltà and Stablum, 1998).

Patients with Parkinson's Disease show difficulties in task switching. For example, in a task in which a colored geometric figure is presented and the participant has to make a discrimination response based on one dimension (e.g., shape: triangle or circle) or another (e.g., color: blue or green), there is an RT cost associated with a dimension shift across trials (e.g., shape $\rightarrow$ color). This cost is abnormally high for individuals with Parkinson's Disease, suggesting an impairment in the ability to switch sets (Hayes et al., 1998). 
The frontal lobes and cortico-striatal loops are made up of a multitude of heterogeneous areas, each with a unique structural and physiological organization. This complexity is matched at the level of information processing, where a collection of loosely integrated processes receives the name of 'executive system'. It is possible that different executive processes might be implemented by separate brain structures. More generally, it is possible to speculate that the brain areas implementing the mental operations currently described as executive function might interact with each other, but might also have unique aspects. As described earlier, this is the case for the orienting and the vigilance networks, and there is no reason to believe that the executive network should be an exception.

\section{Transmitters}

As described earlier, patients with traumatic brain injury are impaired in a myriad of executive functions. These patients, in addition to frontal lobe damage, have diffuse axonal injury, which disrupts the dopaminergic input to the frontal cortex. Schizophrenia and attention deficit disorder, which can be thought of as pathologies of the executive system, seem to involve a dysregulation of the mesocorticolimbic pathway that provides dopaminergic modulation to the anterior cingulate (Casey, in press; Swanson, Castellanos, Murias, LaHoste, \& Kennedy, 1998). Executive dysfunction in Parkinson's Disease is linked to a disruption in the cortico-striatal loop resulting from dopamine deficiency in the striatum, but also to dopamine depletion in the mesocortical pathway, which projects to medial and prefrontal areas.

These findings are consistent with the role of dopamine in executive processes, probably via modulation of the frontal areas. Strong support for this hypothesis comes from a rehabilitation study in which the dopamine agonist bromocriptine was administered to patients with traumatic brain injury who had lesions in prefrontal areas. Bromocriptine improved executive tasks, such as the Stroop task, the Wisconsin Card Sorting Test, and dual tasks (McDowell, Whyte, \& D’Esposito, 1998). Importantly, working memory tasks without an executive demand did not benefit from the therapy, suggesting that the benefit was specific to executive processes.

\section{CONCLUSIONS}

The field of neuropsychology is undergoing a major shift in emphasis as the method and data of neuroimaging begin to be incorporated. In the area of attention, this has involved a greater integration of theory with practice and an increased emphasis on rehabilitation efforts as a means of testing theories as well as providing services to patients.

In this paper, we have emphasized theoretical ideas about attentional networks arising from early imaging studies (Posner \& Petersen, 1990). Newer ideas regarding competition among elements within neural systems (Desimone \& Duncan, 1995) and the role of motor structures (Rizzolatti \& Craighero, 1998) can also be helpful in the design of rehabilitation methods. In the absence of any single comprehensive theory, it is useful to have the perspective of different views when seeking to design the most appropriate methods of rehabilitation. All of these views emphasize anatomical considerations in understanding the nature of deficits. In this article we have tried to illustrate how a variety of disorders that produce orienting problems may be examined within a common anatomical perspective.

Neuroimaging techniques offer new and exciting ways to modify and enhance rehabilitation methods. We can expect improvements in sensitivity, resolution, and time course as new technologies and new adaptations of older methods become available. It is now possible to follow the effects of training, drug, or other therapeutic interventions with structural or functional MRIs, allowing researchers to observe changes that might be induced by the intervention. The use of high-density scalp electrical recording can elucidate changes in the time course of various anatomical areas that might occur as a result of the intervention. The integration of ideas about modulation from transmitter systems with the anatomy of cortical computations can help us understand the relationship between organic brain injury, psychopathologies, and developmental 
abnormalities. As we come to a deeper understanding of the mechanisms of rehabilitation (Robertson, 1999; Robertson \& Murre, 1999), previously competing approaches to rehabilitation may become integrated within an overall strategy of fostering brain recovery.

\section{REFERENCES}

Allport, D.A. (1993). Attention and control: Have we been asking the wrong questions? A critical review of 25 years. In D.E. Meyer \& S. Kornblum (Eds.), Attention and performance XIV (pp. 183-218). Cambridge, MA: MIT Press.

Andersen, R.A., Brotchie, P.R., \& Mazzoni, P. (1992). Evidence for the lateral intraparietal area as the parietal eye field. Current Opinion in Neurobiology, 2, 840-6.

Ashbridge, E., Walsh, V., \& Cowey, A. (1997). Temporal aspects of visual search studied by transcranial magnetic stimulation. Neuropsychologia, 35, 11211131.

Aston-Jones, G., Rajkowski, J., Kubiak, P., \& Alexinsky, T. (1994). Locus coeruleus neurons in monkey are selectively activated by attended cues in a vigilance task. Journal of Neuroscience, 14, 4467.

Badgaiyan, R.D., \& Posner, M.I. (1998). Mapping the cingulate cortex in response selection and monitoring. Neuroimage, 7, 255-260.

Barkley, R.A. (1998). Attention-deficit hyperactivity disorder. Scientific American, 279, 66-71.

Belin, P., McAdams, S., Smith, B., Savel, S., Thivard, L., Samson, S., \& Samson, Y. (1998). The functional anatomy of sound intensity discrimination. The Journal of Neuroscience, 18, 6388-6394.

Buck, B.H., Black, S.E., Behrmann, M., Caldwell, C., \& Bronskill, M.J. (1997). Spatial- and object-based attentional deficits in Alzheimer's disease: Relationship to HMPAO-SPECT measures of parietal perfusion. Brain, 120, 1229-1244.

Bush, G., Luu, P., \& Posner, M.I. (2000). Cognitive and emotional influences in anterior cingulate cortex. Trends in Cognitive Science, 4, 215-222.

Bush, G., Whalen, P.J., Rosen, B.R., Jenike, M.A., McInerney, S.C., \& Rauch, S.L. (1998). The counting Stroop: An interference task specialized for functional neuroimaging-validation study with functional MRI. Human Brain Mapping, 6, 270-282.

Butter, C. M., Kirsch, N. L., \& Reeves, G. (1990). The effect of lateralized stimuli on unilateral spatial neglect following right hemisphere lesions. Restorative Neurology and Neuroscience, 2, 39-46.

Cappa, S.F., Sterzi, R., Vallar, G., \& Bisiach, E. (1987). Remission of hemineglect and anosognosia during vestibular stimulation. Neuropsychologia, 25, 775782.

Carter, C.S., Botvinick, M.M., \& Cohen, J.D. (1999) The contribution of the anterior cingulate cortex to executive processes in cognition. Reviews in the Neurosciences, 10, 49-57.

Casey, B.J. (in press) Disruption and inhibitory control in developmental disorders: A mechanistic model of implicated fronto-striatal circuitry. In J.L. McClelland \& S. Siegler (Eds.), Mechanisms of Cognitive Development: The 29th Carnegie Conference. Hillsdale, NJ: Erlbaum.

Casey, B.J., Trainor, R.J., Orendi, J.L., Schubert, A.B., Nystrom. L.E., Giedd, J.N., Castellanos, F.X., Haxby, J.V., Noll, D.C., Cohen, J.D., Forman, S.D., Dahl, R.E., \& Rapoport, J.L. (1997). A developmental functional MRI study of prefrontal activation during performance of a go-no-go task. Journal of Cognitive Neuroscience, 9, 835-847

Casey, B.J., Trainor, R., Giedd, J., Vauss, Y., Vaituzis, C.K., Hamburger, S., Kozuch, P. \& Rapoport, J.L. (1997). The role of the anterior cingulate in automatic and controlled processes: A developmental neuroanatomical study. Developmental Psychobiology, 3, 61-69.

Chelazzi, L. \& Corbetta, M. (2000). Cortical mechanisms of visuospatial attention in the primate brain. In M.S. Gazzaniga (Ed.), The cognitive neurosciences, vol. 2. Cambridge, MA: MIT Press.

Chiba, A.A., Bushnell, P.J., Oshiro, W.M., \& Gallagher, M. (1999). Selective removal of cholinergic neurons in the basal forebrain alters cued target detection. Neuroreport, 10, 3119-3123.

Clark, C.R., Geffen, G.M, \& Geffen, L.B. (1989). Catecholamines and covert orientation of attention in humans. Neuropsychologia, 27, 131-139.

Cohen, J.D., Romero, R.D., Servan-Schreiber, D., \& Farah, M.J. (1994). Mechanisms of spatial attention: The relation of macrostructure to microstructure in parietal neglect. Journal of Cognitive Neuroscience, 6, 377-383.

Cohen, R.M., Semple, W.E., Gross, M., Holcomb, H.H. (1988). Functional localization of sustained attention: Comparison to sensory stimulation in the absence of instruction. Neuropsychiatry, Neuropsychology, \& Behavioral Neurology, 1, 3-20.

Corbetta, M. (1998). Frontoparietal cortical networks for directing attention and the eye to visual locations: Identical, independent, or overlapping neural systems? Proceedings of the National Academy of Science, 95, 831-838.

Corbetta, M., Miezin, F.M., Dobmeyer, S., Shulman, G.L., \& Petersen, S.E. (1991). Selective and divided attention during visual discriminations of shape, color, and speed: Functional anatomy by positron emission tomography. Journal of Neuroscience, $11,2383-2402$. 
Corbetta, M., Miezin, F.M., Shulman, G.L., \& Petersen, S.E. (1993). A PET study of visuospatial attention. Journal of Neuroscience, 13, 1202-1226.

Coull, J.T., \& Nobre, A.C. (1998). Where and when to pay attention: The neural systems for directing attention to spatial locations and to time intervals as revealed by both positron emission tomography and functional magnetic resonance imaging. Journal of Neuroscience, 18, 7426-7435.

Coull, J.T., Frith, C.D., Frackowiak, R.S.J., \& Grasby, P.M. (1996). A fronto-parietal network for rapid visual information processing: A PET study of sustained attention and working memory. Neuropsychologia, 34, 1085-95.

Coull, J.T., Robbins, T.W., Middleton, H.C., \& Sahakian, B.J. (1995). Clonidine and diazepam have differential effects on tests of attention and learning. Psychopharmacology, 120, 322-332.

Davidson, M.C. \& Marrocco, R.T. (2000). Local infusion of scopolamine into intraparietal cortex alters covert orienting in rhesus monkeys. Journal of Neurophysiology, 83, 1536-1549.

Desimone, R., \& Duncan, J. (1995). Neural mechanisms of selective visual attention. Annual Review of Neuroscience, 18, 193-222.

Devinsky, O., Morrell, M.J., \& Vogt, B.A. (1995). Contributions of anterior cingulate cortex to behaviour. Brain, 118, 279-306.

Duncan, J. (1986). Disorganization of behavior after frontal lobe damage. Cognitive Neuropsychology, 3, 271-290.

Early, T.S., Posner, M.I., Reiman, E.M., \& Raichle, M.I. (1989a). Hyperactivity of the left palido-striatal projection. Psychiatric Development, 2, 85-108.

Early, T.S., Posner, M.I., Reiman, E.M., \& Raichle, M.I. (1989b). Left striato-pallidal hyperactivity in schizophrenia. Psychiatric Development, 2, 109121.

Farah, M. (1994). Neuropsychological inference with an interactive brain: A critique of the "locality" assumption. Behavioral and Brain Sciences, 17, 43-104.

Fernandez-Duque, D., \& Posner, M.I. (1997). Relating the mechanisms of orienting and alerting. Neuropsychologia, 35, 477-486.

Foldi, N.S., Jutagir, R., Davidoff, D., \& Gould, T. (1992). Selective attention skills in Alzheimer's disease: Performance on graded cancellation tests varying in density and complexity. Journal of Gerontology, 47, 146-153.

Frith, C. (1992). The neuropsychology of schizophrenia. Hillsdale NJ: Lawrence Erlbaum.

Greenwood, P.M., Parasuraman, R., \& Alexander, G.E. (1997). Controlling the focus of spatial attention during visual search: Effects of advanced ageing and Alzheimer's disease. Neuropsychology, 11, 312.
Guitton, B., Bouchtel, \& Douglas (1985). Frontal lobe lesions in man cause difficulties in suppressing reflexive glances and in generating goal-directed saccades. Experimental Brain Research, 58, 455472.

Gur, R.E., \& Pearlson, G.D. (1993). Neuroimaging in schizophrenia research. Schizophrenia Bulletin, 19, 337-353.

Halligan, P.W., Manning, L., \& Marshall, J.C. (1991). Hemispheric activation vs. spatio-motor cueing in visual neglect: A case study. Neuropsychologia, 29, 165-176.

Hayes, A.E., Davidson, M.C., Keele, S.W., \& Rafal, R.D. (1998). Toward a functional analysis of the basal ganglia. Journal of Cognitive Neuroscience, 10, 178-198.

Hillyard, S.W., \& Anllo-Vento, L. (1998). Event related potentials in the study of visual selective attention. Proceedings of the National Academy of Sciences of the United States of America, 95, 781-787.

Humphreys, G.W., \& Riddoch, J. (1993). Interactive attentional systems in unilateral visual neglect. In I.H. Robertson \& J. Marshall (Eds.), Unilateral Neglect (pp. 139-168). Hillsdale, NJ: Erlbaum.

Kinsbourne, M. (1977). Hemineglect and hemisphere rivalry. Advances in Neurology, 18, 41-49.

Kinsella, G.J. (1998). Assessment of attention following traumatic brain injury. Neurological Rehabilitation, 8, 351-375.

Köhler, S., Black, S.E., Sinden, M., Szekely, C., Kidron, D., Parker, J.L., Foster, J.K., Moscovitch, M., Winocur, G., Szalai, J.P., \& Bronskil, M.J. (1998). Memory impairments associated with hippocampal versus parahippocampal-gyrus atrophy: An MR volumetry study in Alzheimer's disease. Neuropsychologia, 36, 901-914.

LaBerge, D. (1995). Attentional processing: The brain's art of mindfulness. Cambridge, MA: Harvard University Press.

Làdavas, E. (1993). Shifts of attention in patients with visual neglect. In I.H. Robertson, \& Marshal, J.C. (Eds.), Unilateral neglect: Clinical and experimental studies. Hove (U.K.): Erlbaum.

Làdavas, E., Menghini, G., \& Umiltà, C. (1994). A rehabilitation study of hemispatial neglect. Cognitive Neuropsychology, 11, 75-95.

Làdavas, E., Pretronio, A., \& Umiltà, C. (1990). The deployment of visual attention in the intact field of hemineglect patients. Cortex, 26, 307-317.

Làdavas, E., Zeloni, G., Zaccara, G., \& Gangemi, P. (1997). Eye movements and orienting of attention in patients with visual neglect. Journal of Cognitive Neuroscience, 9, 67-74.

Lawrence, A.D., \& Sahakian, B.J. (1995). Alzheimer's disease, attention, and the cholinergic system. $\mathrm{Alz}$ heimer's Disease and Associated Disorders, 9, 4349. 
Le, T.H., Pardo, J.V., \& Hu, X. (1998). 4 T-fMRI study of nonspatial shifting of selective attention: Cerebellar and parietal contributions. Journal of Neurophysiology, 79, 1535-1548.

Luck, S.J., Fan, S., \& Hillyard, S.A. (1993) Attentionrelated modulation of sensory evked brain activity $\mathrm{n}$ a visual search task. Journal of Cognitive Neuroscience, 5, 188-195.

Luu, P., Collins, P., \& Tucker, D.M. (2000). Mood, personality and self-monitoring: Negative affect and emotionality are related to frontal lobe mechanisms of error detection. Journal of Experimental Psychology: General, 129, 1-18.

Luu, P., Flaisch, T., \& Tucker, D.M. (2000). Medial frontal cortex in action monitoring. Journal of Neuroscience, 20, 464-469.

Marrocco, R.T., \& Davidson, M.C. (1998). Neurochemistry of attention. In R. Parasuraman (Ed.), The Attentive Brain (pp. 35-50). Cambridge, MA: MIT Press.

Maruff, P., Currie, J., Hay, D., McArthur-Jackson, C., \& Malone, V. (1995). Asymmetries in the covert orienting of visual attention in schizophrenia. $\mathrm{Neu}$ ropsychologia, 31, 1205-1223.

Maunsell, J.H.R. (1995). The brain's visual world: Representation of visual targets in cerebral cortex. Science, 260, 764-768.

Mazzoni, P., Bracewell, R.M., Barash, S., \& Andersen, R.A. (1996). Motor intention activity in the macaque's lateral intraparietal area: I. Dissociation of motor plan from sensory memory. Journal of Neurophysiology, 76, 1439-1456.

McDowell, S., Whyte, J., \& D'Esposito, M. (1998). Differential effect of dopaminergic agonist on prefrontal function in traumatic brain injury patients. Brain, 121, 1155-1164.

Morrow, L.A., \& Ratcliff, G. (1987). Attentional mechanisms in clinical neglect. Journal of Clinical and Experimental Neuropsychology, 9, 74-75.

Nebes, R.D. \& Brady, C.B. (1989). Focused and divided attention in Alzheimer's disease. Cortex, 25, 305-315.

Nebes, R.D., \& Brady, C.B. (1993). Phasic and tonic alertness in Alzheimer's disease. Cortex, 29, 77-90.

Nobre, A.C., Gitelman, D.R., Sebestyen, G.N., Meyer, J., Frackowiak, R.S.J., Frith, C.D., \& Mesulam, M.M. (1997). Functional localization of the system for visuospatial attention using positron emission tomography. Brain, 120, 515-533.

Norman, D.A., \& Shallice, T. (1986). Attention to action. Willed and automatic control of behavior. In R.J. Davidson, G.E. Schwartz, \& D. Shaper (Eds.), Consciousness and self regulation (pp. 117). New York: Plenum Press.

Nudo, R.J., Wise, B.M., SiFuentes, F., \& Milliken, G.W. (1996). Neural substrates for the effects of rehabilitative training on motor recovery after ischemic infarct. Science, 272, 1754-1791.
Parasuraman, R., \& Greenwood, P.M. (1998). Selective attention in aging and dementia. In R. Parasuraman (Ed.), The attentive brain (pp. 711-733). Cambridge, MA: MIT Press.

Parasuraman, R., Greenwood, P.M., Haxby, J.B., \& Grady, C.L. (1992). Visuospatial attention in dementia of the Alzheimer's type. Brain, 115, 711-733.

Pardo, J.V., Fox, P.T., \& Raichle, M.E. (1991). Localization of a human system for sustained attention by positron emission tomography. Nature, 349, 61-64.

Pardo, P.J., Knesevich, M.A., Vogler, G.P., Pardo, J.V., Towne, B., Cloninger, C.R., \& Posner, M.I. (2000). Genetic and state variables of neurocognitive dysfunction in schizophrenia: A twin study. Schizophrenia Bulletin, 26, 459-477.

Pascual-Leone, A., Gomez-Tortosa, E., Grafman, J., \& Alway, D. (1994). Induction of visual extinction by rapid-rate transcranial magnetic stimulation of parietal lobe. Neurology, 44, 494-498.

Paus, T., Zatorre, R.J., Hofle, N., \& Caramanos, Z. (1997). Time-related changes in neural systems underlying attention and arousal during the performance of an auditory vigilance task. Journal of Cognitive Neuroscience, 9, 392-408.

Pizzamiglio, L., Frasca, R., Guariglia, C., Incoccia, C., \& Antonucci, G. (1990). Effect of optokinetic stimulation in patients with visual neglect. Cortex, 26, 535-540.

Posner, M.I. (1988). Structures and functions of selective attention. In T. Boll \& B. Bryant (Eds.), Master lectures in clinical neuropsychology and brain function: Research, measurement, and practice (pp. 171-202). Washington, DC: American Psychological Association.

Posner, M.I., \& DiGirolamo, G.J. (1998). Conflict, target detection, and cognitive control. In R. Parasuraman (Ed.), The attentive brain (pp. 401-424). Cambridge: MIT Press.

Posner, M.I., Early, T.S., Reiman, E., Pardo, P.J., \& Dhawan, M. (1988). Asymmetries in hemispheric control of attention in schizophrenia. Archives of General Psychiatry, 45, 814-821.

Posner, M.I., \& Gilbert, C.D. (1999). Attention and primary visual cortex. Proceedings of the National Academy of Sciences of the United States of America, 96, 2585-2587

Posner, M.I., \& Petersen, S.E. (1990). The attention system of the human brain. Annual Review of Neuroscience, 13, 25-42.

Posner, M.I., \& Raichle, M.E. (1994). Images of mind. New York: Scientific American Library.

Posner, M.I., \& Rothbart, M.K. (1998). Attention, selfregulation and consciousness. Philosophical Transactions of the Philosophical Society of London Series B. Biological Sciences, 353, 1915-1927.

Rafal, R. (1998). Neglect. In R. Parasuraman (Ed.), The attentive brain (pp. 711-733). Cambridge, MA: MIT Press. 
Rainville, P., Duncan, G.H., Price, D.D., Carrier, B., \& Bushnell, M.C. (1997). Pain affect encoded in human anterior cingulate cortex but not somatosensory cortex. Science, $277,968-970$.

Rivaud, S., Muri, R. M., Gaymard, B., Vermersch, A. I. \& Pierrot-Deseilligny, C. (1994). Eye movement disorders after frontal eye lesions in humans. Experimental Brain Research, 102, 110-120.

Rizzolatti, G., \& Craighero, L. (1998). Spatial attention: Mechanisms and theories. In M. Sabourin, F. Craik, \& M. Robert (Eds.), Advances in Psychological Science, vol. 2 (pp. 171-197). Have UK: Psychological Press.

Ro, T., Henik, A., Machado, L. \& Rafal, R.D. (1997). Transcranial magnetic stimulation of the prefrontal cortex delays contralateral endogenous saccades. Journal of Cognitive Neuroscience, 9, 433-440.

Robertson, I.H. (1999). Cognitive rehabilitation: Attention and neglect. Trends in Cognitive Science, 3, 385-393.

Robertson, I.H., \& North, N. (1994). One hand is better than two: Motor extinction of left hand advantage in unilateral neglect. Neuropsychologia, 32, 1-11.

Robertson, I.H., \& North, N. (1993). Active and passive activation of left limbs: Influence on visual and sensory neglect. Neuropsychologia, 31, 293-300.

Robertson, I.H., Halligan, P.W. \& Marshal, J.C. (1993). Prospects for the rehabilitation of unilateral neglect. In I.H. Robertson \& J.C. Marshal (Eds.), Unilateral neglect: Clinical and experimental studies. Hove (U.K.): Erlbaum.

Robertson, I.H., Manly, T., Andrade, J., Baddeley, B.T., \& Yiend, J. (1997). “Ooops!”: Performance correlates of everyday attentional failures in traumatic brain injured and normal subjects. Neuropsychologia, 35, 747-758.

Robertson, I.H., North, N., \& Geggie, C. (1992). Spatio-motor cueing in unilateral neglect: Three single case studies of its therapeutic effectiveness. Journal of Neurology, Neurosurgery and Psychiatry, 55, 799-805.

Robertson, I.H., Tegnér, R., Tham, K., Lo, A., \& Nimmo-Smith, I. (1995). Sustained attention training for unilateral neglect: Theoretical and rehabilitation implications. Journal of Clinical and Experimental Neuropsychology, 17, 416-430.

Robertson, I.H., Mattingley, J.B., Rorden, C., \& Driver, J. (1998). Phasic alerting of neglect patients overcomes their spatial deficit in visual awareness. Nature, 395, 169-172.

Robertson, I.H., \& Murre, J.M.J. (1999) Rehabilitation of brain damage: Brain plasticity and principles of guided recovery. Psychological Bulletin, 125, 544575.

Robertson, L. (1998) Visualspatial attention and parietal function: Their role in object perception. In R. Parasuraman (Ed.), The attentive brain (pp. 257278). Cambridge, MA: MIT Press.
Rogers, R.D., \& Monsell, S. (1995). The cost of predictable switch between simple cognitive tasks. Journal of Experimental Psychology: General, 124, 207-231.

Rueckert, L., \& Grafman, J. (1996). Sustained attention deficits in patients with right frontal lesions. Neuropsychologia, 34, 953-963.

Schall, J.D. (1995). Neuronal basis of saccadic target selection. Reviews in the Neurosciences, 6, 63-85.

Schwartz, M.F. (1987). Focal cognitive deficits in dementia of the Alzheimer's type. Neuropsychology, $1,27-35$.

Scinto, L.F.M., Daffner, K.R., Castro, L., Weintraub, S., Varvrik, M., \& Mesulam, M.M. (1994). Impairment of spatially directed attention in patients with probable Alzheimer's disease as measured by eye movements. Archives of Neurology, 51, 682-688.

Seyal, M., Ro, T. \& Rafal, R. (1995). Increased sensitivity to ipsilateral cutaneous stimuli following transcranial magnetic stimulation of the parietal lobe. Annals of Neurology, 38, 264-267.

Smith, A. \& Nutt, D. (1996). Noradrenaline and attention lapses. Nature, 380, 291.

Sohlberg, M.M., McLaughlin, K.A., Pavese, A., Heidrich, A., \& Posner, M.I. (2000). Evaluation of attention process training in persons with acquired brain injury. Journal of Clinical and Experimental Neuropsychology. 22, 656-676.

Strum, W., Willmes, K., Orgass, B., \& Hartje, W. (1997). Do specific attention effects need specific training? Neurological Rehabilitation, 12, 81-103.

Swanson, J., Castellanos, F.X., Murias, M., LaHoste, G., \& Kennedy, J. (1998). Cognitive neuroscience of attention deficit hyperactivity disorder and hyperkinetic disorder. Current Opinion in Neurobiology, 8, 263-271.

Taub, E., Miller, N.E., Novack, T.A., Cook, E.W., Fleming, W.C., Nepomuceno, C.S., Connell, J.S., \& Crago, J.E. (1993). Technique to improve chronic motor deficit after stroke. Archives of Physical Medicine and Rehabilitation, 74, 347-354.

Toga, A.W., \& Mazziotta, J.C. (1994). Brain mapping: The methods. New York: Academic Press.

Umiltà, C., \& Stablum, F. (1998). Control processes explored by the study of closed-head-injury patients. In G. Mazzoni \& T.O. Nelson (Eds.), Metacognition and cognitive neuropsychology: Monitoring and control processes. Mahwah, NJ: Lawrence Erlbaum Associates.

Umiltà, C., Nicoletti, R., Simion, F., Tagliabue, M.E., \& Bagnara, S. (1992). The cost of a strategy. European Journal of Cognitive Psychology, 4, 21-40.

Vallar, G., Sterzi, R., Bottini, G., Cappa, S., \& Rusconi, M.L. (1990). Temporary remission of left hemianaesthesia after vestibular stimulation: A sensory neglect phenomenon. Cortex, 26, 123-131.

Voytko, M.L., Olton, D.S., Richardson, R.T., Gorman, L.K., Tobin, J.R., \& Price, D.L. (1994). Basal forebrain lesions in monkeys disrupt attention but not 
learning and memory. Journal of Neuroscience, 14, 167-186.

Walsh, V., \& Cowey, A. (1998). Magnetic stimulation studies of visual cognition. Trends in Neuroscience, 2, 103-110.

West, R.L. (1996). An application of prefrontal cortex function theory to cognitive aging. Psychological Bulletin, 120, 272-292.
Wilkins, A.J., Shallice, T., \& McCarthy, R. (1987). Frontal lesions and sustained attention. Neuropsychologia, 25, 359-366.

Witte, E.A., \& Marrocco, R.T. (1997). Alteration of brain noradrenergic activity in rhesus monkeys affects the alerting component of covert orienting. Psychopharmacology, 132, 315-323. 